\title{
UPGRADING OF THE SINGLE POINT LASER VIBROMETER INTO A LASER SCANNING VIBROMETER
}

The paper proposes a construction design of measuring device for fast and reliable non-contact modal analysis of object surfaces which cannot be measured using classical methods. The design combines conventional non-contact laser interferometer with an active optics module to be able to measure multiple points in a rapid sequence. The paper briefly discusses all the key constructional components and describes in detail the system functional layout. It also introduces the experimental measurement of a HV transformer under operation to demonstrate the system functionality.

Keywords: modal analysis, laser, doppler, vibrometry, measurement, prototype

\section{Introduction}

The method of Laser Doppler Vibrometry (LDV) has been widely used in medical $[1,2]$ or engineering applications involving the non-contact vibration [3-7] and can be also adopted for electrical engineering, especially when contact with the measured surface is not possible, such as measuring machines under operation, measuring of hot surfaces or surfaces which are under high voltage operation $[8,9]$.

However, since most single point LDV systems are only capable of sensing data for one point at a time, performing modal analysis to obtain an operational deflection shape tend to be quite long process. This problem is even more difficult for bigger structures or for structures with low natural frequencies, such as aircraft, space structures or civil structures, which would require more measurement points and excessively longer testing time if a single point LDV were used [10-12].

There are several types of laser-scanning vibrometers available on the market that can measure multiple points simultaneously, but their cost is too high. Only a few literature sources deal with its own vibrometer design whereas the most relevant work to this issue has been published in [13]. Here, the authors introduced a design of three-dimensional vibration measurement system consisting of one laser scanning vibrometer, one CCO camera and one laser scanner. Though it can measure the shape and vibration of the tested object at the same time, it is still expensive.
This paper describes the unique device developed to measure vibration for fast and reliable modal analysis of facial surfaces. Measurement of vibration via LDV has been used as the instrument of choice when contactless measurement and measurement with high spatial detail is required. The proposed vibrometer construction allows us to redirect the laser beam via computer controlled mirrors to acquire data for predefined matrix of measurement points. Moreover, it upgrades the single-point vibrometer into the fast programmable $2 \mathrm{D}$ fullfield vibration scanner [14, 15].

\section{Construction design}

The proposed measuring device, seen in Figure 1, exploits the benefits of a functional connection of the conventional noncontact laser interferometer (PDV 100) and the active optics with the fast steering mirrors. The interferometer generates laser beam which is sequentially redirected via the computer controlled mirrors in a predefined pattern. This solution enables us to perform multi-point measurements in a rapid sequence, having no back effect on the tested object. In addition, it will considerably simplify either the operational deflection shape (ODS) or the modal analysis of complicated 3D objects. The influence of the varying deflection angle of the laser beam pointing the measurement locations can be reduced using the proper software

\footnotetext{
* ${ }^{1}$ Tomas Kavalir, ${ }^{1}$ Michal Krizek, ${ }^{1}$ Jiri Sika, ${ }^{2}$ Vladimir Kindl

${ }^{1}$ Regional Technological Institute, Faculty of Mechanical Engineering, University of West Bohemia, Pilsen, Czech Republic

${ }^{2}$ Regional Innovation Centre for Electrical Engineering, Faculty of Electrical Engineering, University of West Bohemia, Pilsen, Czech Republic

E-mail: vkindl@kev.zcu.cz
} 


\section{kOMNIKocie}
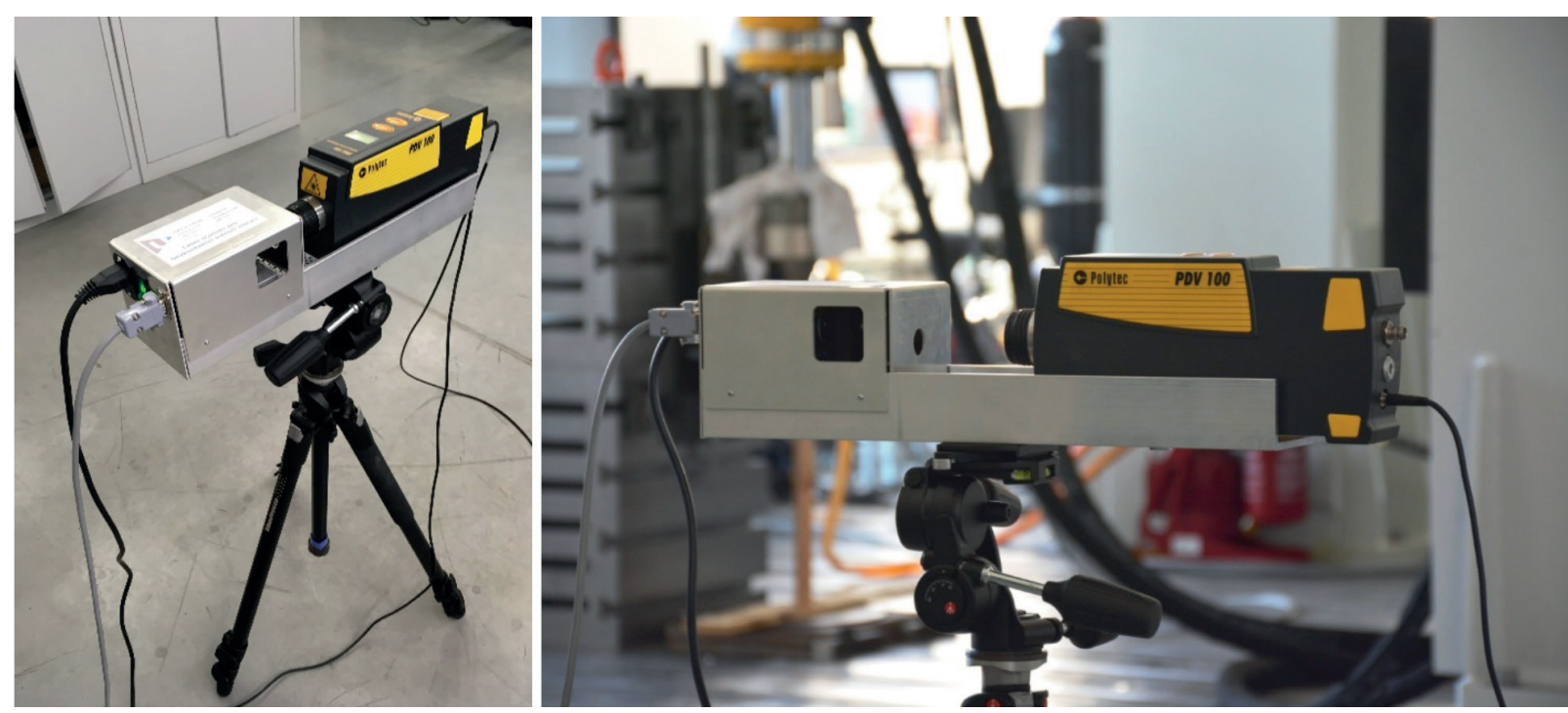

Figure 1 LDV with XY scan head
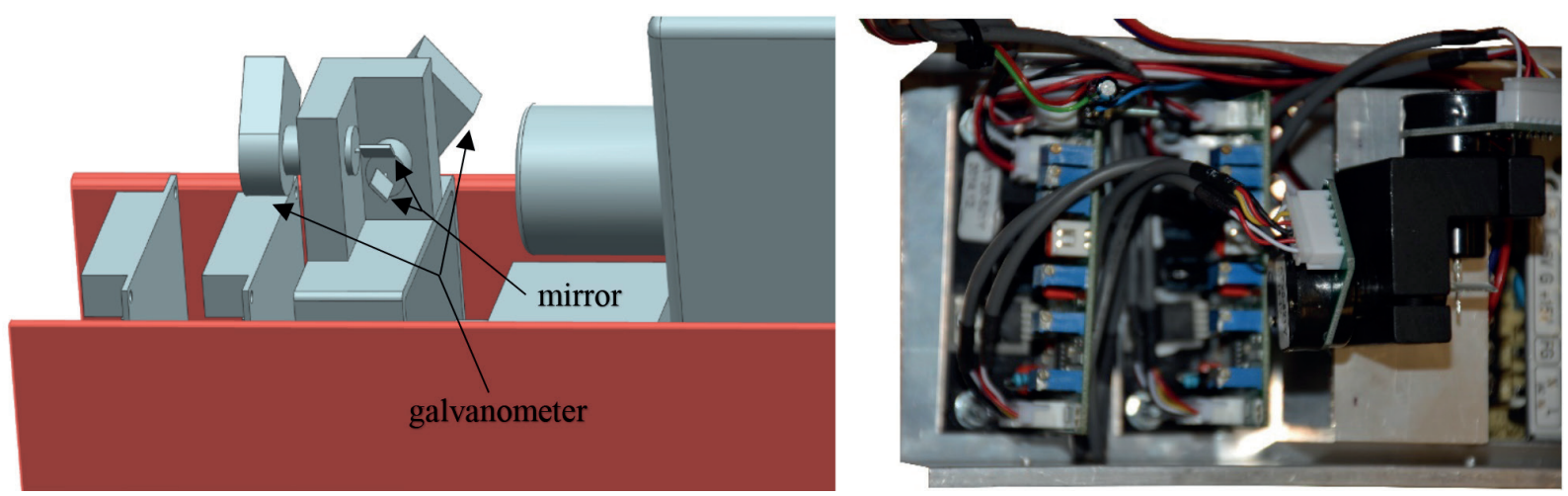

Figure 2 Detailed 3D mechanical model of the measuring system with the LDV (left), inside view of the mirrors with galvanometer and control electronics (right)

compensation, but if the testing object is far enough, it would be neglected.

The laser deflecting module (see Figure 2) is based on the principle of galvanometer with special mirrors mounted on its axes. In this case, we adapted the mechanism of the low-cost X-Y GALVO scanner, commonly used in the field of entertainment electronics for sweeping and rendering the laser beam patterns. In order to prevent any laser beam disturbance, the mirrors should provide high reflectivity and low roughness. The mechanism is driven by electronics, sometimes called DC servo, giving us very fast and accurate dynamic response of the deflection module measured in both axes. All the components are assembled together on a mutual chassis made up of structural $\mathrm{AL}$ profiles, forming a compact device suitable for practical application.

Figure 3 shows an input control console, based on a singleboard microcomputer, using an intelligent graphical display to show either the relevant communication data or the main systems settings. The controller is based on the Arduino Esplora programmable array, including control buttons, joystick for the laser beam control and local storage to store programmable data for further re-editing or resetting the measurement sequence. The control console is mounted into a compact plastic chassis printed by a $3 \mathrm{D}$ printer.

The measured data are processed using the PULSE ${ }^{\mathrm{TM}}$ analyses system developed by BK Company [16]. The system is extended with our own software routine to perform the modal analyses and includes measurement card with accessories that processes the signals from the LDV and the auxiliary reference sensor. The overall specification of developed measuring device may be listed in Table 1.

\section{Experiment}

For the functional demonstration, the operational deflection shape of a $22 \mathrm{kV}$ transformer, with $1000 \mathrm{kVA}$ of rated power, was made. The transformer was partially loaded during the test and was located in the HV transformer cell behind a barrier (see 


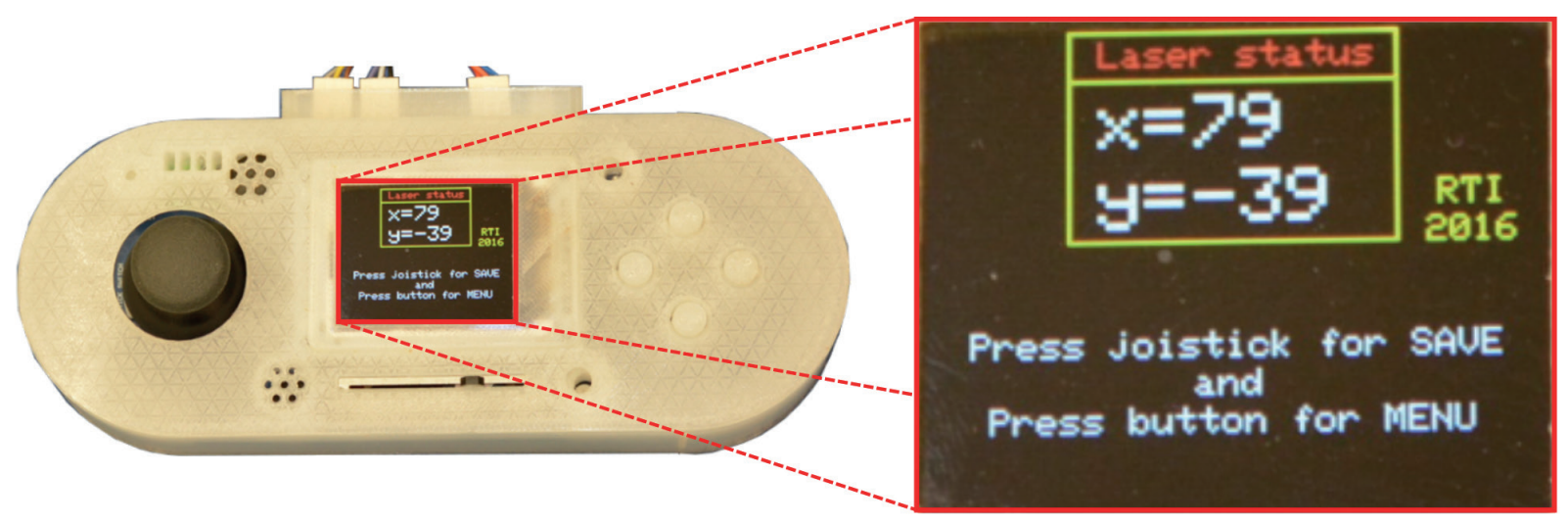

Figure 3 The control console with Arduino Esplora for XY scan head
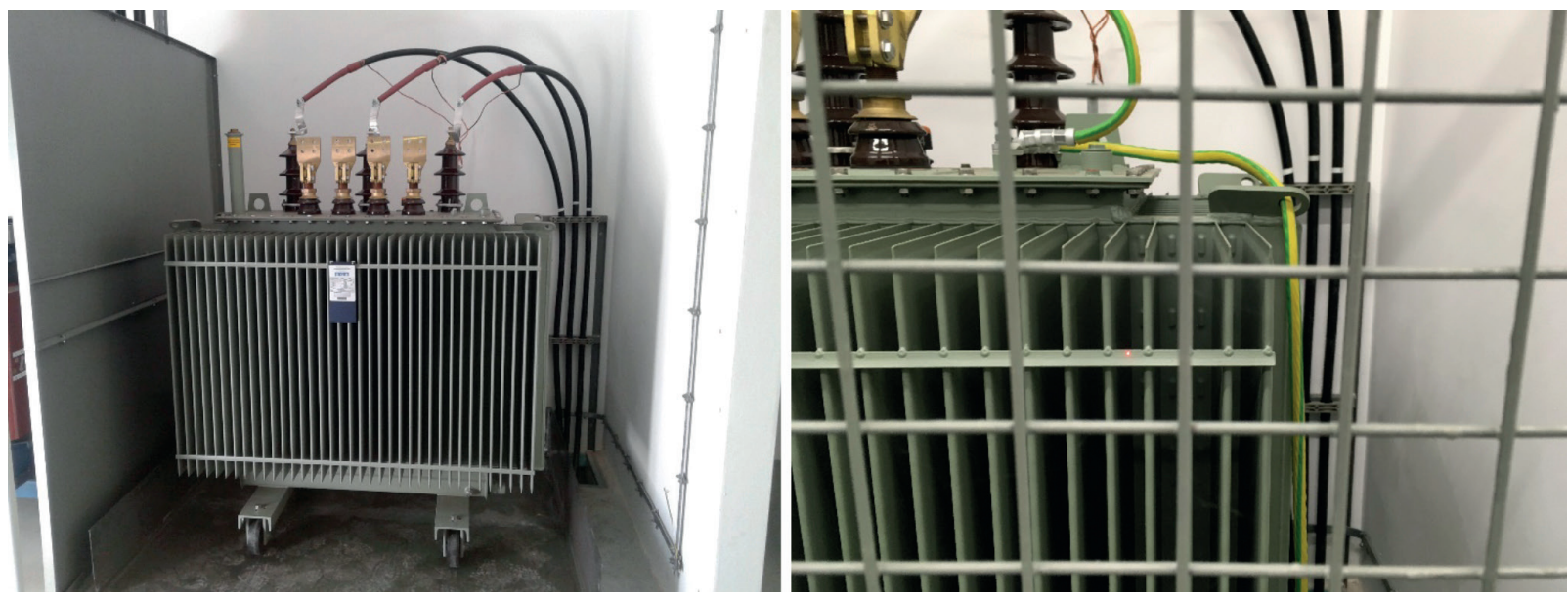

Figure 4 Tested HV transformer; without the safety barrier (left), measuring points programming (right)

Table 1 Proposed device specification

\begin{tabular}{lll}
\hline Parameter & Values & Additional Notes \\
\hline Input resistance & $200 \mathrm{k} \Omega$ & differential \\
Signal input voltage & $\pm 5 \mathrm{~V}$ & \\
Input voltage requirements & $+15 \mathrm{~V} / 1.0 \mathrm{~A}$ and $-15 \mathrm{~V} / 0.6 \mathrm{~A}$ & \\
Operating temperature range & $0 \div 50^{\circ} \mathrm{C}$ & \\
Operational optical angle & \pm 30 degrees & max \\
Scanner speed & $>15 \mathrm{kpps}$ & $30 \mathrm{kpps}, \pm 20^{\circ}$ optical \\
Mirror dimensions (width, height, thickness) & $7 \mathrm{~mm}, 11 \mathrm{~mm}, 0.6 \mathrm{~mm}$ & wide wave-length \\
\hline
\end{tabular}

Figure 4). Our goal was to demonstrate the strong advantage of the proposed system laying in possibility to measure under conditions when the testing operator has no direct access to the tested object. Thus, it is particularly relevant to the application where there is no possibility to measure using classical contact methods (like accelerometers) due to the high voltage, high temperature or other possible reasons.

The testing procedure may be understood from Figure 5. The required $x$ - and $y$ - positions of the laser beam are considered as inputs to the CPU (Arduino Esplora). This command is set using the joystick and then it is sampled by an 8-bit A/D transducer. Data are further processed by the predefined algorithm, but they could be additionally modified or manipulated. Up to 256 x 256 pixels (measuring points) can be uploaded into the internal or external storage (micro SD card). Then, the sequence of stored points can be recalled and executed by defined scanning speed. At the output of the CPU, two signals corresponding to the $x$ - and $y$ - positions are generated by the PWM modulation. Since the control unit for the galvanometer requires a DC signal fluctuating in the range of $0 \div 5 \mathrm{~V}$, a low-pass filter is applied at the output of 


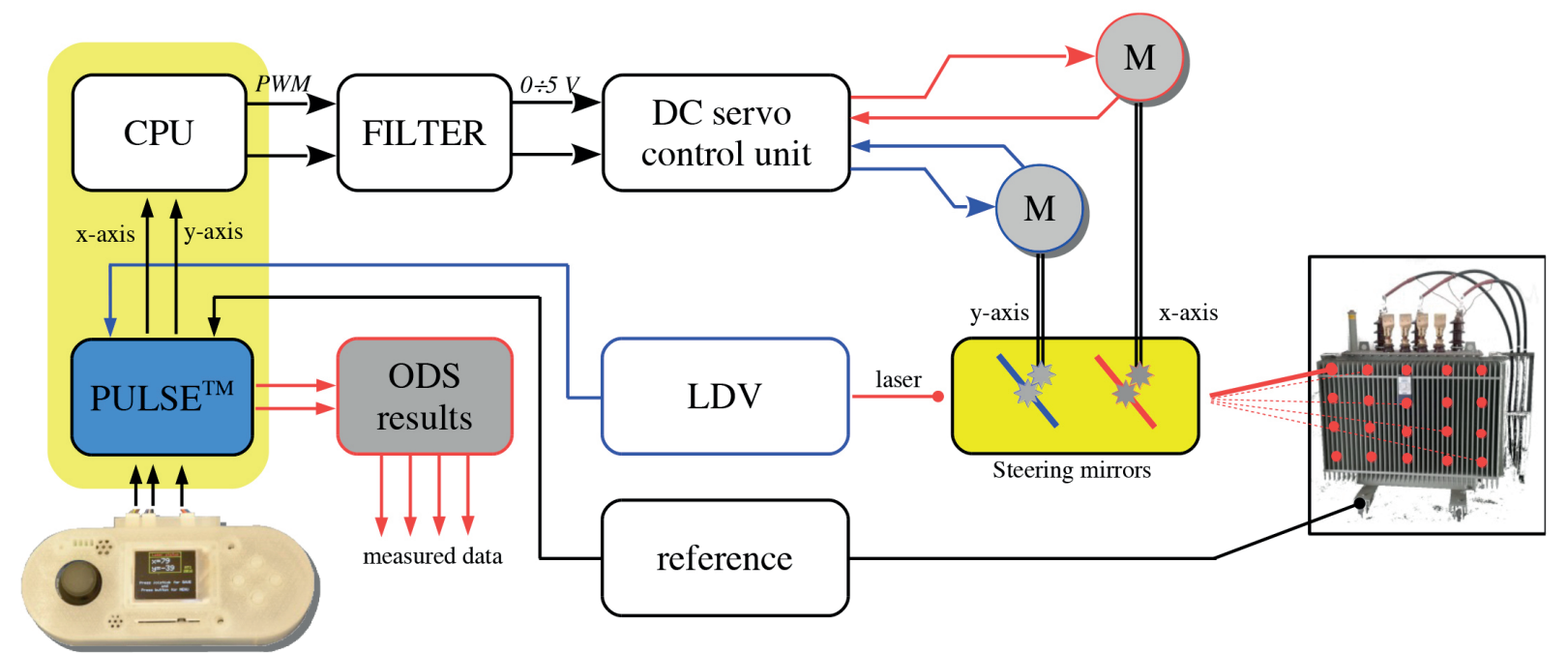

Figure 5 Block diagram of the measuring procedure

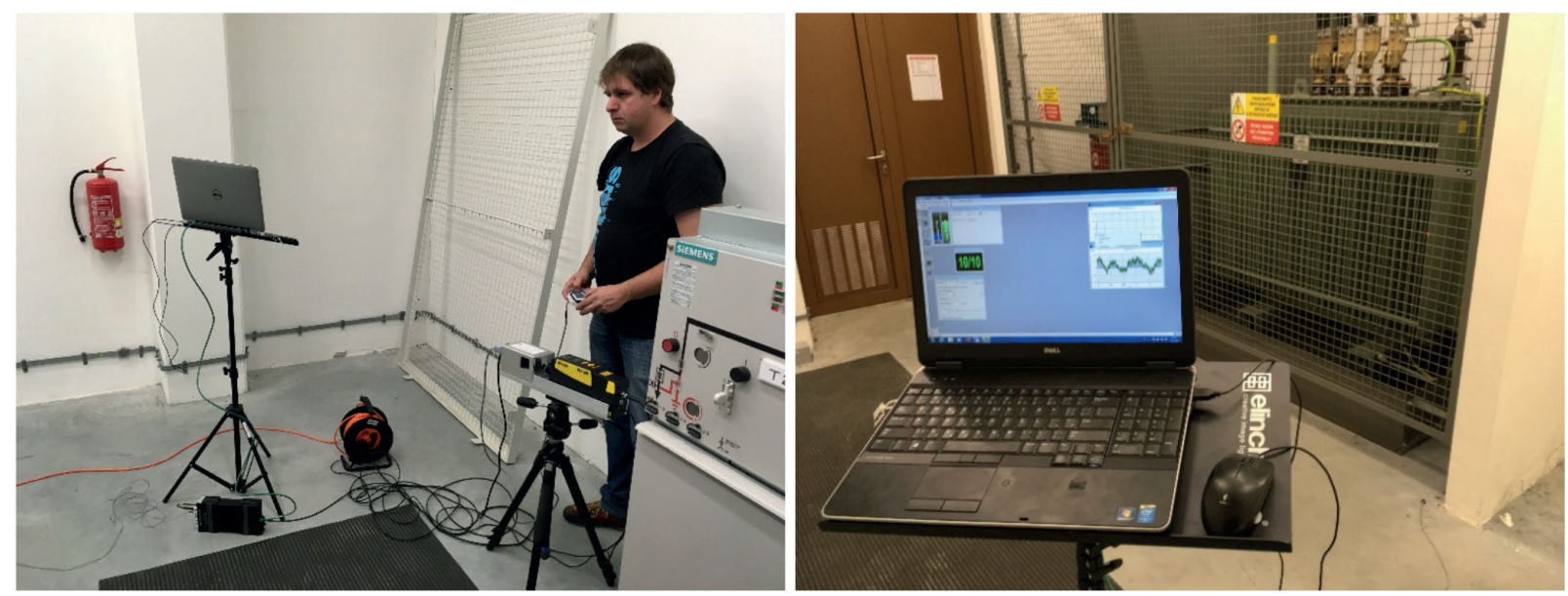

Figure 6 Experimental setup; the programming procedure (left), calibration (right)

the CPU. This regulation is highly accurate and quick enough to provide precise mirror adjustments according to set commands.

The measuring mechanism is driven by the PULSE ${ }^{\mathrm{TM}}$ system extended with our own SW routine for data processing. It continuously collects data from the LDV and the reference sensor, needed for the proper system synchronization. Based on these data, the operational deflection shape, corresponding to the evaluated vibration, is calculated.

Figure 6 shows the situation during the measurement. On the left-hand side, the procedure of programming is seen, the righthand side shows the preparatory measurement and the system calibration.

The resulting vibration map measured on the transformer is seen in Figure 7. The results show that the proposed constructive solution of the testing device is fully functional and it is also suitable for the non-contact vibration sensing. As a consequence, the device upgrades a common single point vibrometer into the full-filed vibrometer, which can visualize either the Eigen modes or the operating deflection shapes of any tested object.

\section{Conclusion}

The experimental measurement has proven good operability of the proposed laser-scanning vibrometer even in an industry application. Due to the fact that the design combines conventional non-contact laser interferometer with a low-cost X-Y GALVO scanner, primary designated to the field of entertainment electronics, we have achieved significant financial savings while maintaining very good operational usability. The vibrometer can measure multiple points in a rapid sequence and hence it will find its purpose especially when measuring either the Eigen modes or the operating deflection shapes. Moreover, the measurement is non-contact and therefore it has no feedback effect on 


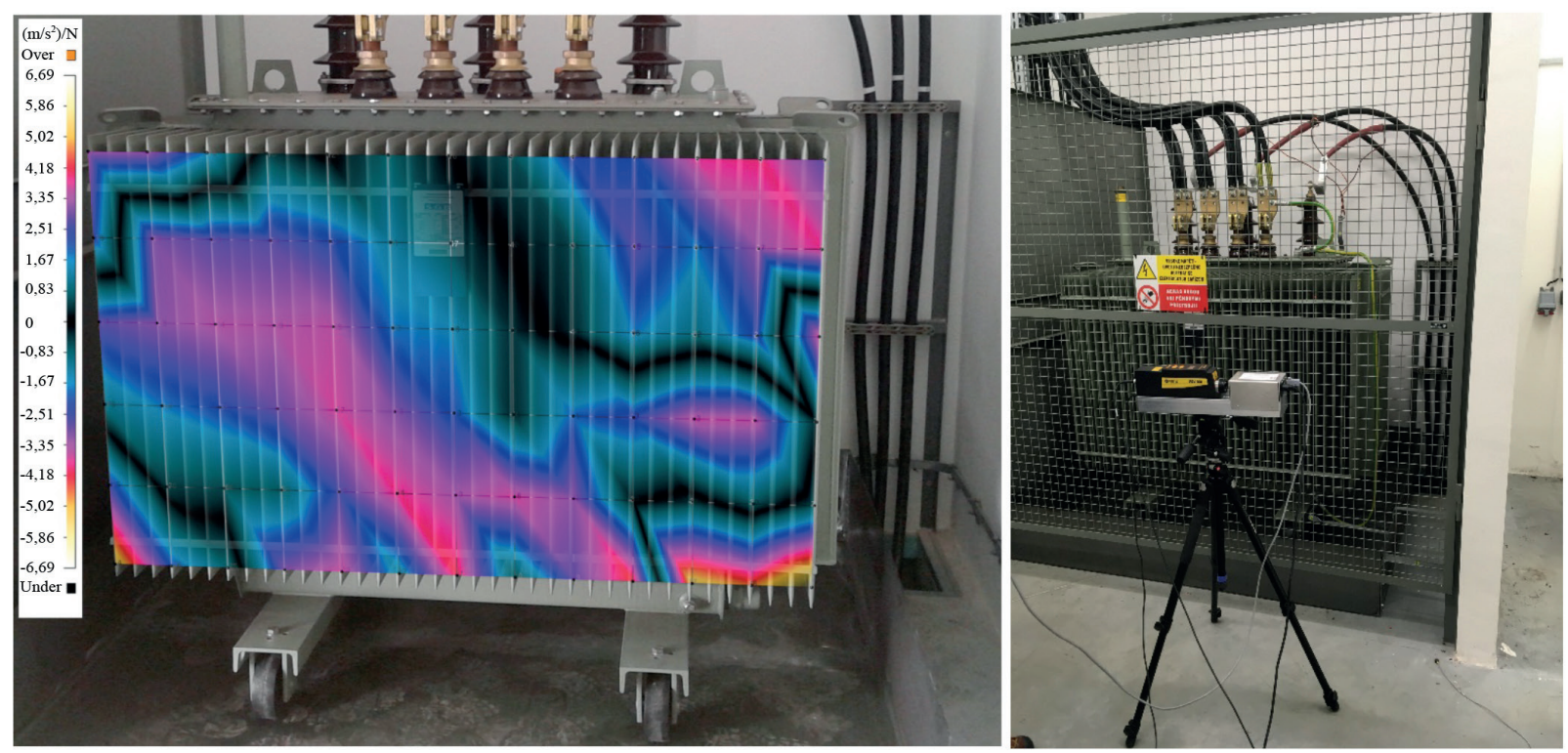

Figure 7 Measured ODS results mapped onto the transformer

a tested object. This may also be beneficial for testing objects operating under high voltage, high temperature or being otherwise inaccessible. The bottle neck seems to be ensuring proper surfaces reflection together with manual focusing of laser beam between measured points. There is also the need of dedicated analysis hardware and software tool to display results. For field measurement it is recommend a dust protection because dust can damage the functionality of the mirror based optical system.

\section{Acknowledgements}

The present contribution has been prepared under project LO1502 "Development of the Regional Technological Institute" under the auspices of the National Sustainability Programme I of the Ministry of Education of the Czech Republic aimed to support research, experimental development and innovation. It was also supported by the Ministry of Education, Youth and Sports of the Czech Republic under the RICE - New Technologies and Concepts for Smart Industrial Systems, project No. LO1607.

\section{References}

[1] CASACCIA, S., SCALISE, L., CASACANDITElla, L., TOMASINI, E. P., ROHRBAUGH, J. W.: Non-Contact Assessment of Muscle Contraction: Laser Doppler Myography. Proceedings of IEEE International Symposium on Medical Measurements and Applications (MeMeA), Italy, 610-615, 2015.

[2] SCALISE, L., ERCOLI, I., MARCHIONNI, P., TOMASINI, E. P.: Measurement of Respiration Rate in Preterm Infants by Laser Doppler Vibrometry. Proceedings of IEEE International Symposium on Medical Measurements and Applications, Italy, 657-661, 2011.

[3] KANDRAC, J., FRIVALDSKY, M., PRAZENICA, M., SIMONOVA, A.: Design and Verification of Proposed Operation Modes of LLC Converter. Elektronika ir Elektrotechnika/ Electronics and Electrical Engineering, 18(8), 27-30, 2012.

[4] TORCIANTI, B., CRISTALLI, C., VASS, J.: Non-Contact Measurement for Mechanical Fault Detection in Production Line. Proceedings of IEEE International Symposium on Diagnostics for Electric Machines, Power Electronics and Drives, Poland, 297. 301, 2007.

[5] SPANIK, P., SEDO, J., DRGONA, P., FRIVALDSKY, M.: Real Time Harmonic Analysis of Recuperative Current through Utilization of Digital Measuring Equipment. Elektronika ir Elektrotechnika/ Electronics and Electrical Engineering, 19(5), 33-38, 2013.

[6] HUANG, Y. H., MA, C. C.: Experimental Measurements and Finite Element Analysis of the Coupled Vibrational Characteristics of Piezoelectric Shells. IEEE Transactions on Ultrasonics, Ferroelectrics, and Frequency Control, 59(4), 785-798, 2012.

[7] MALlYA, A. N., RAMAMURTHY, P. C. : Mechanical Actuation of Conducting Polymer in the Presence of Organic Vapor Stimulus. IEEE Sensors Journal, 17(11), 3391-3397, 2017. 


\section{KonNIlkCle}

[8] Sugimoto, T., AKAMATSU, R., UTAGAWA, N., TSUJinO, S.: Study on Non Contact Acoustic Imaging Method for Non Destructive Inspection Using SLDV and LRAD. Proceedings of IEEE International Ultrasonics Symposium, USA, 744-747, 2011.

[9] KOSCELNIK, J., FRIVALDSKY, M., PRAZENICA, M., MAZGUT, R.: A Review of Multi-Elements Resonant Converters Topologies. Proceedings of 10th IEEE International Conference ELEKTRO 2014, Slovakia, 312-317, 2014.

[10] LAURENTIU-MARIAN, A., ALEXANDROS, R.: A Theoretical Approach Based on the Correlation of Unified Data for Different Analyzing Techniques Used in the Modern Investigation of Artworks. Proceedings of Developments in E-systems Engineering, England, 89-92, 2010.

[11] REITEN, M. T., WRIGHT, R. G.: Laser Doppler Vibrometry Use in Detecting Faulty Printed Circuit Boards. Proceedings of IEEE AUTOTESTCON, USA, 33-36, 2008.

[12] KIM, D., SONG, H., KHALIL, H., LEE, J., WANG, S., PARK, K.: Corrections to "3-D Vibration Measurement Using a Single Laser Scanning Vibrometer by Moving to Three Different Locations” [Aug 14 2028-2033]. IEEE Transactions on Instrumentation and Measurement, 63(10), 2504-2504, 2014.

[13] AMBRozINSKI, L., SPYTEK, J., DZIEDZIECH, K., PIECZONKA, L., STASZEWSKI, W. J.: Damage Detection in PlateLike Structures Based on Mode-Conversion Sensing With 3D Laser Vibrometer. Proceedings of IEEE International Ultrasonics Symposium (IUS), USA, 1-4, 2017.

[14] YOON, H., SONG, H., PARK, K.: A Phase-Shift Laser Scanner Based on a Time-Counting Method for High Linearity Performance. Review of Scientific Instruments, 82(7), 075108, 2011.

[15] KIM, D., PARK, K.: Development of a Three-Dimensional Vibration Measurement System Using a Single Laser Scanning Vibrometer and Laser Scanner. Proceedings of 13th International Conference on Control, Automation and Systems (ICCAS 2013), South Korea, 1378-1380, 2013.

[16] BK Company: Pulse [online]. Available: https://www.bksv.com/media/doc/bu0229.pdf. 